\title{
Perceptual Significance of Inharmonicity and Spectral Envelope in the Piano Bass Range
}

\section{Alexandre Galembo}

Ryerson University

\section{Anders Askenfelt}

Royal Institute of Technology

\section{Lola L. Cuddy}

Queen's University

\section{Frank A. Russo}

Queen's University

\section{Please Cite:}

Galembo, A., Askenfelt, A., Cuddy, L. L., \& Russo, F. A. (2004). Ergonomics action research II: A Framework for Integrating Human Factors into WorkSystem Design. Acta Acustica united with Acustica, 90(3), 528-536.

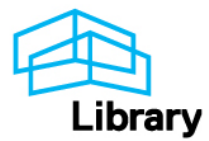




\title{
Perceptual relevance of inharmonicity and spectral envelope in the piano bass range
}

\author{
Alexander Galembo \\ Setchenov Institute of Evolutionary Physiology and Biochemistry, Russian Academy of \\ Sciences, Thorez pr. 44, St. Petersburg, 194223 Russia. \\ Anders Askenfelt \\ Dept. of Speech, Music and Hearing, Royal Institute of Technology, SE-100 44 Stockholm, \\ Sweden.
}

Lola L. Cuddy \& Frank A. Russo

Dept. of Psychology, Queen's University, Kingston ON K7L 3N6, Canada.

\begin{abstract}
Professionals consider the differences in the timbre of bass tones between large grand pianos and small uprights as significant. By tradition this difference has been attributed mainly to lower inharmonicity in grand pianos, due to longer bass strings. In this study, the importance of the spectral envelope, representing the dynamic balance between high-frequency and lowfrequency energy in the spectrum, is contrasted against the importance of the level of inharmonicity. Results from two listening tests indicate that the inharmonicity is less important than the spectrum bandwidth in determining the timbre of piano bass tones.
\end{abstract}




\section{INTRODUCTION}

Even a well-made upright piano of high quality is not considered appropriate for concert performances. A large grand piano is deemed to surpass an upright in all respects. This is due to several factors only few of which are clearly acoustical [1]. With regard to timbre in the bass, a grand piano is said to have "a brighter and mellower timbre," "more powerful and projecting sound," while in comparison, bass tones of a small upright might be referred to as "muffled, dull and inexpressive" [2-4].

These perceived differences could be attributed to differences in the design. The most important differences in design pertaining to the favoured acoustical output of the concert grand piano are longer strings and a larger soundboard. Longer strings make it possible to keep the inharmonicity in the bass range lower. A larger soundboard will have lower mode frequencies in general, and a higher mode density. As a ball-park value, the lowest soundboard mode frequency of an upright would be around $100 \mathrm{~Hz}$, and for a large grand some $30-40 \mathrm{~Hz}$ lower. The modes below, let's say $100 \mathrm{~Hz}$, will give support for the fundamental and lower overtones in the two lowest octaves of the piano compass. The higher mode density would give a less irregular spectrum envelope for higher partials with less pronounced dips and valleys in the spectrum envelope.

By tradition, the effect of inharmonicity has been assumed the most important factor influencing tone quality in the bass range. After having conducted the first measurements on the inharmonicity in real pianos, Schuck \& Young [5] hypothesized that the lower inharmonicity in the bass range (due to longer strings) explains why musicians prefer the tone quality of a grand piano over that of an upright. Later, Fletcher, Blackham and Stratton investigated the perception of synthesized piano tones, and claimed that the inharmonicity was highly important for the "peculiar quality known as piano quality, namely, the live-ness or warmth of a tone" [6].

Since Fletcher et al.'s statement, which seemed to suggest a strong perceptual basis for the hypothesis of Shuck and Young, it has became common to attribute the primary difference in perceived quality of bass tones of small vs. large pianos to the differences in string inharmonicity (see e.g. [7-8]). Further experiments with synthesis of piano tones [9-11] have strengthened this point, showing that the inharmonicity strongly influences the timbre of a multi-component tone.

In spite of many investigations on the physics of the string-soundboard interaction [12-20] there is still not enough experimental results available to assess the individual effects of string inharmonicity and soundboard properties on the timbral differences between small and large pianos. There is no easy way of separating the perceptual influence due to the effects of longer strings from the influence of a larger soundboard. In this study, two experiments are reported which give a starting point for further investigations of this important aspect of piano design. 


\subsection{String inharmonicity and number of partials vs. spectral bandwidth}

The classical formula describing the stretching of the frequencies of the transverse modes is (see e.g. [21])

$$
f_{n}=n f_{1}^{o} \sqrt{1+n^{2} B}
$$

where

$n \quad$ partial number

$f_{1}{ }^{o} \quad$ fundamental frequency of a flexible string

$f_{n} \quad$ frequency of partial $n$

$B \quad$ inharmonicity coefficient, set by the string material, dimensions, and tension (tuning)

An expression for the inharmonicity coefficient $B$, valid for plain strings and approximately correct for wrapped strings [8], is

$$
B=\left(\frac{\pi}{8}\right)^{2}\left(\frac{E}{\rho F}\right)\left(\frac{d}{f_{1}^{o} L^{2}}\right)^{2}
$$

where

$$
\begin{array}{ll}
E & \text { Young's modulus } \\
\rho & \text { density of plain string materiel (core material of wrapped string) } \\
d & \text { string (core) diameter } \\
L & \text { speaking length } \\
F & \text { loading factor } \quad F=\frac{\mu_{\text {core }}+\mu_{w r}}{\mu_{\text {core }}}=\frac{\mu_{s t r}}{\mu_{\text {core }}}=M_{s t r} / L \\
\mu_{c o r e}, \mu_{w r}, \mu_{s t r} & \begin{array}{l}
\text { linear density of the core, wrapping and whole string } \\
M_{s t r}
\end{array} \\
& \text { mass of speaking length of string }
\end{array}
$$

The simple relations between inharmonicity and string properties, in which the string length takes a prominent role, have inspired researchers to try to demonstrate the importance of inharmonicity on timbre by synthesizing piano-like tones for which only the inharmonicity coefficient $B$ is varied $[6,9,10]$. Although such demonstrations appear quite convincing, they overlook one perceptually important parameter, which covaries with inharmonicity. That is the frequency range occupied by prominent partials in the spectrum, henceforth the spectral bandwidth [22].

In the bass section (about two lowest octaves) the inharmonicity coefficient $B$ may differ a factor 5 between the same keys of an upright and grand piano [23]. The total variation range of $B$ in this register has been reported as $60-160 \cdot 10^{-6}$ in a large grand, and $130-570 \cdot 10^{-6}$ 
in a small upright. The spectral bandwidths for two tones with such widely differing $B^{\prime} \mathrm{s}$ but with the same number of partials will differ largely. For a low piano bass tone the number of prominent partials $N$ in a range of $-60 \mathrm{~dB}$ below the strongest partial may well be above 100 at high dynamic levels (see Fig. 1). Even in a 40-dB range below the strongest partial there will be as many as $70-80$ partials. The spectrum of a bass tone with high inharmonicity ( $B$ $\approx 600 \cdot 10^{-6}$ ) and about 100 partials will extend to a frequency more than twice as high as for a harmonic tone, adding a full octave to the spectral bandwidth (see Fig. 2).

An increase of the spectral bandwidth changes the balance between high and low frequency energy and influences the brightness (sharpness) of the tone [24-27]. It seems possible that similar timbral differences could be obtained by just increasing the number of overtones without change of the inharmonicity coefficient. If so, the inharmonicity factor plays only a secondary role. To disambiguate the simultaneous influence of these two factors, we designed two experiments aimed at resolving the individual effects of inharmonicity and spectral bandwidth on timbre. Synthesized harmonic and inharmonic tones, as well as recorded real piano tones, were used.

\section{EXPERIMENT 1: Synthesized tones}

\subsection{Synthesis}

Twelve piano-like tones with nominal pitch $\mathrm{E}_{1}\left(f_{o}=41.2 \mathrm{~Hz}\right)$ which varied in spectral bandwidth and inharmonicity were synthesized. Four values of spectral bandwidth $W_{i}(i=1$, $2,3,4)$, ranging from 2.5 to $1.4 \mathrm{kHz}$, were combined with three levels of inharmonicity $B_{k}$ $(k=1,2,3)$ see Table 1 . The bandwidths represented typical values in real pianos, as outlined in the following paragraphs. The magnitude of the three values of inharmonicity coefficient $B$ represented: (1) a harmonic spectrum, $B_{1}=0$; (2) an inharmonicity close to the minimal value for $\mathrm{E}_{1}$ measured in grand pianos, $B_{2}=60 \cdot 10^{-6}$; and (3) an inharmonicity close to the maximum values observed for $\mathrm{E}_{1}$ measured in poor upright pianos, $B_{3}=600 \cdot 10^{-6}$ [23]. The number of partials necessary to fill a spectral bandwidth $W_{i}$ at inharmonicity level $B_{k}$ was denoted $N_{i, k}$. As the string inharmonicity stretches the partials according to Eq.(1), fewer partials are required for filling a fixed spectral bandwidth the higher the inharmonicity coefficient.

The widest bandwidth $W_{1}(2513 \mathrm{~Hz})$ corresponded to the spectrum of an $\mathrm{E}_{1}$-tone with about 60 prominent partials, which is representative of the conditions immediately after note onset. The narrowest bandwidth $W_{4}(1442 \mathrm{~Hz})$ corresponded to the spectrum after $1-3 \mathrm{~s}$ of decay. The intermediate steps, $W_{2}(1978 \mathrm{~Hz})$ and $W_{3}(1648 \mathrm{~Hz})$, were chosen in such a manner that it was possible to achieve them in two ways; (1) by changing the inharmonicity coefficient without changing number of partials, and (2) by changing the number of partials without significantly changing the inharmonicity coefficient. This overlap allowed a convenient illustration of the individual effects of $W$ and $B$, respectively, on perceived differences in timbre in the listening test. 
The first principle using a constant number of partials was applied as to make $N_{i, 3}=N_{i+1,1}$ (marked in bold in Table 1). The harmonic tones $W_{2,1}, W_{3,1}$, and $W_{4,1}$ were thus generated with the same number of partials as the high-inharmonicity tone of the preceding larger bandwidth, using $N=48,40$ and 35, respectively. The second principle was used to generate the low-inharmonicity tones $W_{2,2}$ and $W_{3,2}$ using approximately the same $B$ as for $W_{1,2}$ and $W_{4,2}\left(60,68,75,52 \cdot 10^{-6}\right.$; numbers in italics refer to $W_{2,2}$ and $\left.W_{3,2}\right)$, but with intermediate values of $N(56,45,38,34)$.

The specific numbers were calculated as follows. Suppose a tone with a harmonic spectrum $(B=0)$ containing $N$ harmonics of the fundamental $f_{l}{ }^{\circ}$. Now we want to reduce the number of partials to $N_{x}<N$ while keeping both the highest and the lowest partial frequencies - and consequently the spectral bandwidth - unchanged. This is achieved by selecting a $B_{x} \neq 0$, and finding a proper lowering of the (unstretched) fundamental $f_{l}{ }^{o}$ to a new value $f_{l x}{ }^{o}$. The new values are defined by

$$
\left\{\begin{array}{l}
N f_{1}^{0}=N_{x} f_{1 x}^{0} \sqrt{1+B_{x} N_{x}^{2}} \\
f_{1}^{0}=f_{1 x}^{0} \sqrt{1+B_{x}^{2}}
\end{array}\right.
$$

that gives

$$
\begin{aligned}
& B_{\mathrm{x}}=\frac{\left(N^{2}-N_{x}^{2}\right)}{\left(N_{x}^{4}-N^{2}\right)} \\
& f_{1 x}^{0}=\frac{f_{1}^{0}}{\sqrt{1+\left(N^{2}-N_{x}^{2}\right) /\left(N_{x}^{4}-N^{2}\right)}}
\end{aligned}
$$

Equation (5) was solved numerically in order to obtain a solution with $B_{x}$ close to the desired value while $N_{x}$ being an integer. The resulting sets of $B, N$ and $f_{l}{ }^{o}$ for the 12 tones are given in Table 1. 


\begin{tabular}{|c|c|c|c|c|c|c|c|c|c|c|c|c|}
\hline & \multicolumn{3}{|c|}{$\begin{array}{c}\text { Bandwidth } W_{1} \\
2513 \mathrm{~Hz}\end{array}$} & \multicolumn{3}{|c|}{$\begin{array}{c}\text { Bandwidth } W_{2} \\
1978 \mathrm{~Hz}\end{array}$} & \multicolumn{3}{|c|}{$\begin{array}{c}\text { Bandwidth } W_{3} \\
1648 \mathrm{~Hz}\end{array}$} & \multicolumn{3}{|c|}{$\begin{array}{c}\text { Bandwidth } W_{4} \\
1442 \mathrm{~Hz}\end{array}$} \\
\hline$K$ & $\begin{array}{c}W_{1,1} \\
1\end{array}$ & $\begin{array}{c}W_{1,2} \\
2\end{array}$ & $\begin{array}{c}W_{1,3} \\
3\end{array}$ & $\begin{array}{c}W_{2,1} \\
1\end{array}$ & $\begin{array}{c}W_{2,2} \\
2\end{array}$ & $\begin{array}{c}W_{2,3} \\
3\end{array}$ & $\begin{array}{c}W_{3,1} \\
1\end{array}$ & $\begin{array}{c}W_{3,2} \\
2\end{array}$ & $\begin{array}{c}W_{3,3} \\
3\end{array}$ & $\begin{array}{c}W_{4,1} \\
1\end{array}$ & $\begin{array}{c}W_{4,2} \\
2\end{array}$ & $\begin{array}{c}W_{4,3} \\
3\end{array}$ \\
\hline$B \times 10^{-6}$ & $\begin{array}{c}\text { HARM } \\
0\end{array}$ & $\begin{array}{c}\text { LOW } \\
60\end{array}$ & $\begin{array}{c}\mathrm{HIGH} \\
267\end{array}$ & $\begin{array}{c}\text { HARM } \\
0\end{array}$ & $\begin{array}{c}\text { LOW } \\
68\end{array}$ & $\begin{array}{c}\mathrm{HIGH} \\
275\end{array}$ & $\begin{array}{c}\text { HARM } \\
0\end{array}$ & $\begin{array}{c}\text { LOW } \\
75\end{array}$ & $\begin{array}{c}\mathrm{HIGH} \\
250\end{array}$ & $\begin{array}{c}\text { HARM } \\
0\end{array}$ & $\begin{array}{c}\text { LOW } \\
52\end{array}$ & $\begin{array}{c}\mathrm{HIGH} \\
286\end{array}$ \\
\hline$N$ & 61 & 56 & 48 & 48 & 45 & 40 & 40 & 38 & 35 & 35 & 34 & 31 \\
\hline $\begin{array}{l}\Delta f_{1}{ }^{\circ} \times 10^{-3} \\
\text { rel } 41.2 \mathrm{~Hz}\end{array}$ & 0 & -1.0 & -5.5 & 0 & -1.4 & -5.7 & 0 & -1.5 & -5.2 & 0 & -1.1 & -5.9 \\
\hline
\end{tabular}

Table 1. Spectral bandwidth $W$, inharmonicity coefficient $B$, number of partials $N$, and (unstretched) fundamental frequency $f_{l}{ }^{o}$ for 12 synthesized piano-like tones. The value for $f_{l}{ }^{o}$ is given as the deviation (in $\mathrm{mHz}$ ) from the nominal fundamental frequency for $\mathrm{E}_{1}=41.200 \mathrm{~Hz}$. The tones in shaded cells (bold) represent cases where different bandwidths were generated by the same number of partials.

Since $N$ must be an integer, it is inevitable to have some freedom in the $B$ values in order reach the four chosen spectral bandwidths. For this reason the $B$ values vary slightly between the four $W$ cases. However, the $B_{2}$ values $\left(60,68,75\right.$, and $\left.52 \cdot 10^{-6}\right)$ remain close to the limits $\left(60-90 \cdot 10^{-6}\right)$, which have been reported as typical for an $\mathrm{E}_{1}$ note in contemporary large grand pianos [23]. Similarly, the $B_{3}$ values $\left(267,275,250\right.$, and $\left.286 \cdot 10^{-6}\right)$ vary essentially within the range $\left(200-280 \cdot 10^{-6}\right)$ typical of the maximum inharmonicity for $\mathrm{E}_{1}$ in contemporary small uprights. By the adjustment of the $B$ values it was possible to keep the spectral bandwidth constant for the three notes corresponding to each $W$ case, the spread being less than $1 \mathrm{~Hz}$.

The tones were generated by additive synthesis on a PC [28] according to the formula

$$
\text { tone }(t)=A \sum_{n=1}^{N} S(n) D(n) G(n) \sin (2 \pi f(n) t+\Phi(n, 0))
$$

where

$$
\begin{array}{ll}
A & \text { gain } \\
t & \text { current time } \\
n & \text { partial number } \\
N & \text { number of partials } \\
S(n)=1-n /(N+1) & \text { slope factor } \\
G(n)=0.3+0.7 \sin ^{2}(2 \pi n / 15) & \text { spectral grouping factor } \\
D(n)=\mathrm{e}^{-t n 2 / 200} & \text { decay factor } \\
f(n)=f_{1}{ }^{o} n\left(1+n^{2} B\right)^{0.5} & \text { frequency of } n \text {-th partial } \\
\Phi(n, 0)=\operatorname{random}[0,2 \pi] & \text { starting phase of the } n \text {-th partial }
\end{array}
$$


The synthesis parameters $A(n), G(n)$, and $D(n)$ were chosen as to mimic the spectral properties of real piano tones as closely as possibly (see Fig. 3). The slope factor $S(n)$ gives a linear spectral slope from the fundamental up to the $N$-th partial. The grouping factor $G(n)$ gives a "formant-like" structure related to the striking point of the hammer with $7-8$ partials in each group, and the decay factor $D(n)$ approximates the characteristic decay of piano tones with progressively faster decay of higher partials. The gain $A$ was used for loudness equalization of the tones (see Sect. 2.2.4 and 3.2.4)

\subsection{Listening test 1}

\subsubsection{Listeners.}

Eight listeners from the Queen's University community were recruited for this test. Three of the listeners were involved in loudness equalization. The remaining five listeners were involved in rating timbral difference. Music training of listeners was assessed by a point system. One point was awarded for each year of private instruction and a half point was awarded for each year of group instruction. Listeners had a minimum of 5 points, a mean of 9.25 points $(\underline{\mathrm{SE}}=1.94)$, and typically had experience with 2 instruments and some continued activity in music beyond casual listening. Thus, listeners may be described as musically trained.

\subsubsection{Stimuli.}

The test tones were the 12 tones in Table 1 described above. The standard tone was the harmonic test tone with maximal spectral bandwidth $\left(W_{l}=2513 \mathrm{~Hz} ; B_{1}=0\right)$. Pairing each test tone with the standard tone created twelve tone-pairs. The duration of the test tones was $1.0 \mathrm{~s}$. Tone-pairs had an inter-tone separation of $0.1 \mathrm{~s}$.

\subsubsection{Apparatus}

A PC Pentium computer running dedicated listening test software controlled the stimulus presentation and collection of responses [29]. Tones were presented using a small computer loudspeaker (Creative CS100), specified to be within $\pm 3 \mathrm{~dB}$ across $20-20000 \mathrm{~Hz}$. The listening tests were conducted in a small office room $(2 \times 3 \mathrm{~m})$ with concrete walls. Consideration of phase response of the loudspeaker presentation has been addressed in [11].

\subsubsection{Loudness equalization.}

All tones were equalized for loudness based on the informal loudness scaling of one expert listener. The standard tone was presented with an intensity of $72 \mathrm{~dB}$ SPL as measured at the position of the listener. The listener was asked to iteratively adjust the amplitude of each test tone until it was equal in loudness with the standard tone. Two other listeners validated that the amplitude-adjusted test tones were equal in loudness.

\subsubsection{Procedure.}

The order of the test tone and standard tone within each pair was counterbalanced, allowing for 24 possible tone-pair combinations. The subjects' task was to rate the perceived timbral difference in each tone-pair between 0 and 1000, using a scroll bar. Each tone-pair combination was rated 5 times, giving 120 trials for each subject. The order of presentation was independently randomized for each subject. 


\subsection{Results.}

Each subjects' test-tone ratings were collapsed across order of presentation and trials to obtain 12 timbral-difference scores. Fig. 4 is a plot of mean timbral-difference scores with reference to each test tone's spectral bandwidth $(W 1-W 4)$ and inharmonicity $(B 1-B 3)$. Examination of Figure 4 indicates that the effect of spectral bandwidth on perceived timbral difference is greater than the effect of inharmonicity.

Two vectors with 12 items each were used to represent the respective levels of each independent variable: spectral bandwidth $(1,2,3,4)$ and inharmonicity $(1,2,3)$. Correlation analyses using each subject's timbral difference scores as the dependent variable confirmed that the effect of spectral bandwidth on perceived timbral difference is greater than the effect of inharmonicity. Specifically, for each subject, the correlation between spectral bandwidth and timbral-difference scores was significant (mean $r[10]=.97, \mathrm{p}<.001$ ), but the correlation between inharmonicity and timbral difference scores was not (mean $r[10]=.07$, $\mathrm{p}>$.1). Moreover, when spectral bandwidth and inharmonicity were regressed on each subject's timbral-difference scores, inharmonicity was never able to account for explained variance beyond that which was accounted for by spectral bandwidth alone.

These findings lead us to conclude that spectral bandwidth, or the energy balance between high-frequency and low-frequency partials contributed to the perception of timbral difference to a greater extent than inharmonicity. Given that the tested levels of spectral bandwidth and inharmonicity span a range that is typical of real pianos, this conclusion has direct implications for perception of timbral differences in real piano tones.

In summary, Listening test 1 indicated that when inharmonicity "competes" with spectral bandwidth per se, spectral bandwidth has the most influence on perceived timbral difference.

\section{Experiment 2: Real piano tones}

\subsection{Hybrid tone}

To provide further support for the result of the previous listening test, experiments were conducted with a synthesized hybrid tone. Tones from a concert grand and a small upright piano, respectively, were used as prototypes for different parameters of the hybrid tone.

The purpose was to discriminate between the spectral envelope, including spectral bandwidth, and inharmonicity factors in the timbre of bass tones of small vs. large pianos. As the most distinct timbral difference was expected to be found in the extreme bass, the lowest tone on the piano was chosen for the experiments.

The hybrid tone had the pitch of $\mathrm{A}_{0}=27.5 \mathrm{~Hz}$ and a duration of $1 \mathrm{~s}$. The inharmonicity $(B=$ $160 \cdot 10^{-6}$ ) was set according to measurements on a concert grand piano (Steinway \& Sons, model $\mathrm{D}, 2.74 \mathrm{~m}$ ), and the spectral envelope, including the evolution during the decay, 
imitated that of a small upright piano (Nordiska Pianofabriken, model Futura, $112 \mathrm{~cm}$ ). It could be noted in passing that the inharmonicity of the upright piano $\left(B=560 \cdot 10^{-6}\right)$ was three times higher than that of the Steinway grand.

The hybrid tone was then synthesized using the same principles as in Experiment 1 (see Eq. 7). The imitation of the spectral envelope of the upright piano and its evolution was based on careful Fourier analyses of the recorded tone. The parameters of the formula were varied from one group of partials to another in order to fit the frequency borders, envelope shape, and evolution with time [cf. 30] The difficulties in setting the parameters were primarily due to the fact that the frequencies of partials in the hybrid tone were completely different from those in the upright prototype, due to the exchanged inharmonicity coefficient. The final tuning of the parameters was to a large extent based on visual inspection of spectra and listening to the resulting tones.

The analysis via synthesis process of ended up by a somewhat simplified imitation of the spectral envelope of the upright tone shown in Fig. 5. The spectral bandwidth was closely reproduced, as well as the main peaks and widths of the "formant" groups in the spectrum. The decay process of the upright tone was reproduced by adjusting the peak values of the main formant groups and the bandwidth of the spectra at 3 time points during the 1-s decay. This gave a reasonable approximation of the evolution of the spectral envelope.

\subsection{Listening test 2}

\subsubsection{Listeners.}

Six listeners from the Queen's University community were recruited for this test. Three of the listeners were involved in the loudness equalization. The remaining three listeners were involved in rating timbral difference. Music training of listeners was assessed by the point system described in Listening Test 1. Listeners had a minimum of 5 points, a mean of 9.38 points $(\mathrm{SE}=1.99)$, and typically had experience with two instruments and some continued activity in music beyond casual listening. Thus, listeners may be described as musically trained.

\subsubsection{Stimuli.}

There were three test tones: (1) an upright prototype tone (from the original recording of the Nordiska piano), (2) a grand prototype tone (from the original recording of the Steinway D), and (3) the hybrid tone. The duration of the test tones was $1.0 \mathrm{~s}$. The hybrid tone was paired with each tone including itself resulting in 3 different tone pairs. Tone-pairs had an intertone duration of $0.1 \mathrm{~s}$.

\subsubsection{Apparatus}

The apparatus was identical to that used in Listening test 1, except that stimuli presentation was over headphones (Sennheiser HD580), specified to be within $\pm 3 \mathrm{~dB}$ across 16 $20000 \mathrm{~Hz}$. Consideration of the phase response of headphone presentations has been addressed in [11]. 


\subsubsection{Loudness equalization.}

All tones were equalized for loudness based on the loudness scaling of three listeners. Given the mix of real and synthesized tones, the method used for loudness scaling was more formal for that used in Listening Test 1. Specifically, on any given trial, the listener was presented with the standard tone followed by a comparison tone, or a comparison tone followed by the standard tone (presentation order was counterbalanced). The listener was asked to make a 3alternative forced choice response (i.e., $1^{\text {st }}$ tone is louder, $2^{\text {nd }}$ tone is louder, or tones possess equal loudness). The $\mathrm{A}_{0}$ tone of the Nordiska upright piano served as the standard tone in all trials. Its sound level as measured at the surface of the headphone was $72 \mathrm{~dB}$. Eleven comparison tones for each alternative (i.e. Steinway and Synthesized) were individually paired with the standard tone. Comparison tones varied in their intensity such that median intensity of comparison tones was equal to that of the standard tone. The selection of an equal-loudness tone (with regard to the standard) for each alternative timbre was made on the basis of the comparison tone that received the greatest proportion of equal-loudness responses.

\subsubsection{Procedure}

The order of tones within each tone-pair was counterbalanced, allowing for 6 possible tonepair combinations. The listeners' task was to scale the perceived timbral difference in each tone-pair combination between 0 and 1000, using a scroll bar [29]. Each tone-pair combination was rated on 5 separate occasions, giving 30 trials for each subject. The order of presentation was independently randomized for each of 3 subjects.

\subsection{Results}

Timbral difference ratings were collapsed across presentations. For each of the three listeners, the timbral difference between the hybrid tone and the upright piano tone was significantly lower than the timbral difference between the hybrid tone and the grand piano tone; $t(5)$ values were $-5.79,-7.90$ and -4.98 (all $p<.01$ ). In Figure 6 , the mean timbral difference between the hybrid tone and the upright tone (mean $=233.8, \mathrm{SD}=178.5)$ may be compared with the mean timbral difference between the hybrid tone and the grand piano tone $($ mean $=690.7, \mathrm{SD}=219.8)$.

The results of Listening test 2 clearly indicate that the timbral difference between the hybrid and the upright was less than the timbral difference between the hybrid and the grand - that is to say, the hybrid sounded closer to the upright. Given that the hybrid imitated the spectral bandwidth of the upright and the inharmonicity of the grand, we can conclude that spectral envelope influenced timbre more than inharmonicity. 


\section{DISCUSSION}

\subsection{The importance of inharmonicity vs. spectral envelope}

Poor tone quality in the bass of small pianos is a problem for piano manufacturers, who would be happy to improve the design. Given the present experimental results, we can conclude that it is not sufficient to design strings with lower inharmonicity in order to improve the sound quality in the bass section of small pianos. The spectral envelope parameters (set by the soundboard properties) and their evolution during the decay are of greater importance. The prominence of inharmonicity in determining the tone quality would thus be lower than generally assumed. This misconception is most probably due to the fact that in real pianos, inharmonicity and spectrum bandwidth covary.

As a matter of fact, the old studies by Schuck and Young [5] and Fletcher et al. [6] - from which the widespread notion of the unique role of inharmonicity as the key quality parameter of piano bass tones has developed - do not give reliable support for the dominance of inharmonicity over other piano design parameters. The Schuck and Young hypothesis was based on comparisons of the measured inharmonicity between several pianos of different size and quality. These pianos differed in inharmonicity, but probably in other respects as well.

Fletcher et al compared recorded and synthesized piano tones and made the classical statement that inharmonicity is necessary to give synthesized bass tones the characteristic timbral "warmth" of natural piano tones. ${ }^{\text {FOOTNOTE } 1}$ However, they did not conclude that inharmonicity is the main quality parameter, not even in the bass range. In more subtle discriminations between pianos other factors may be of a similar or higher importance.

When comparing inharmonic tones by timbre, it is important to remember that the inharmonicity coefficient is not the only - and probably even not the primary - measure of the perceptual effect of progressive stretching of partials in piano-like tones. Important factors are how many inharmonic partials are audible and their relative magnitudes. In this sense, the inharmonicity coefficient and spectral envelope factors interact.

For example, the inharmonicity coefficient of the short treble strings is much higher than that of the bass strings. However, treble tones contain only few overtones, located in a frequency range of low audibility. Therefore, the perceptual effect of the string inharmonicity in the treble range is negligible.

Another consequence of the interaction between inharmonicity coefficient and spectral envelope is related to the evolution of the piano tone. The inharmonicity coefficient does not change with time but the perceptual effect of the stretching of the partials is maximal in the initial portion of the tone. This is due to the progressively faster decay of higher partials which narrows the spectrum over time as illustrated in Fig. 3. 
In all pitch ranges of the piano, the higher order partials deviate farther from the harmonic series, thus detracting more from the strength of the (nominal) pitch of the tone. Further, in bass tones the higher partials fall in a better audible frequency range than the fundamental and low order partials. The effect of inharmonicity coefficient on timbre can therefore be expected to be stronger in bass tones with wider spectra (cf. the large grand and the small upright piano in Fig. 5).

Some recent studies indirectly support our finding that that the timbral role of inharmonicity is overestimated. None of them gives, however, as strong evidence as the present study. Lee $[31,32]$ found in experiments on synthesis of piano-like tones that, with regard to tone quality, it was enough to reproduce the inharmonicity in the attack portion of the tone. He concluded that inharmonicity played an insignificant role for the timbre of the decaying part of the piano tone.

Rocchesso \& Scalcon [33] tried to synthesize naturally sounding piano tones, supposing that for every pitch there exists a frequency above which the inharmonicity does not contribute to the naturalness of the timbre. In the bass, this border was found to rise from about 1700 $\mathrm{Hz}$ for $\mathrm{C}_{1}$ (corresponding to about the $51^{\text {th }}$ partial), to $3800 \mathrm{~Hz}$ for $\mathrm{C}_{3}$ (29 ${ }^{\text {th }}$ partial). It must be mentioned, however, that this study, as well as those by Lee [31, 32], were restricted to auditory classification of instrument group ("piano" - "not piano"), which is not the same as ranking pianos by their tone quality.

Beauchamp, McAdams, and Meneguzzi [34], who analyzed acoustical factors determining the identification of musical instruments, pointed out that spectral irregularity is a more important factor of musical timbre than inharmonicity. Their study did, however, not deal specifically with the piano but other musical instruments, including the clarinet, flute, violin, harpsichord and marimba.

Although the results published by other researchers seem to be in general accordance with our finding that inharmonicity is a secondary factor in determining the difference in timbre between a large grand and small upright piano, we are far from concluding that inharmonicity is irrelevant for the quality of pianos. When basic quality conditions, including the spectral envelope among other things, are met, a difference in inharmonicity may be discriminating. As a parallel example, substituting a fine soundboard of tone wood by a veneer board may not be as noticeable in a small upright as in a large grand.

Some support for the importance of inharmonicity in achieving a high-quality piano tone could be gained from an unsuccessful attempt to use the hybrid-tone approach for synthesizing an acceptable imitation of a grand piano bass tone. Our idea was to combine the "good" spectral envelope of a concert grand piano tone with the "bad" inharmonicity of an upright. The resulting tones were, however, far from a successful imitation of the timbre of any of the two prototypes. Of course, this could be due to the shortcomings in our analysis and synthesis, in particular the level of details. However, one other possible explanation would be that, after all, the synthesis was accurate enough but never heard before - a piano tone with a preferred spectral envelope but high inharmonicity. When the spectral envelope does not give cues in the direction of a poor piano, the influence of inharmonicity may be more clearly exposed, and hence important. 


\subsection{Relations to piano design}

The results reported in this study may be given an approximate interpretation in terms of piano design and technology, partly based on the first author's long experience as a piano design engineer. Table 2 shows the schematic dependence of the two investigated parameters of a piano bass tone (inharmonicity coefficient and spectral envelope) on some major piano design factors.

\begin{tabular}{|l|c|c|}
\hline \multicolumn{1}{|c|}{ INSTRUMENT DESIGN FACTORS } & $\begin{array}{c}\text { INHARMONICITY } \\
\text { COEFFICIENT }\end{array}$ & SPECTRAL ENVELOPE \\
\hline Instrument size & $\mathbf{X}$ & $\mathbf{X}$ \\
\hline String material and design & $\mathbf{X}$ & $\mathbf{X}$ \\
\hline Soundboard material and design & $\mathrm{X}$ & $\mathbf{X}$ \\
\hline Hammer material and design & & $\mathbf{X}$ \\
\hline Lids and case & & $\mathbf{X}$ \\
\hline
\end{tabular}

Table 2. Schematic overview of the influence of piano design factors on inharmonicity coefficient and spectral envelope. The two sizes of crosses suggest the relative importance.

The instrument size restricts both the string scale and soundboard dimensions, thus setting a lower limit for the string inharmonicity in the bass. Also the cut-off in sound radiation at lower frequencies is determined by size.

The string parameters (core and wrapping material and dimensions, the uniformity of the wrapping, etc.) and the string tension influence primarily the inharmonicity of the string modes. As regards the influence on the spectral envelope of the radiated tone, the string parameters seem to play only a minor role, partly explained by their rather limited range of variation in practice.

The soundboard design parameters, setting the mode frequencies and dampings, jointly form a primary factor in the evolution of the spectral envelope of the radiated sound. The design parameters include the shape, dimensions, and material of the soundboard, the way it couples to the strings (downbearing), and the way it is fastened along the perimeter. The soundboard parameters also influence the inharmonicity. This effect relates mainly to the lower partials in the bass range [8], shifting them from the frequencies predicted by the string inharmonicity formula [35]. The perceptual effect of this shift has not been studied so far. 
The striking position of the hammer defines the formant-like grouping of the partials in the spectrum. This grouping is important when the partials are numerous (bass range), but not important when they are few (treble range). On the other hand, in the treble where; (1) the hammer-string contact duration exceeds half a period of the fundamental [36], (2) the hammer strikes the strings very close to the termination at the iron frame (capo d'astro bar), and (3) the soundboard is relatively stiff, the hammer parameters become a dominant factor in determining the spectrum envelope of the radiated tone [37].

The spectral envelope and its evolution strongly depend on the reverberation processes, which starts with the soundboard, a distributed radiator, and continue within the piano case, thus depending on design of the case and lid.

As seen in Table 2, the spectral envelope and its evaluation depend on all listed design factors, while the inharmonicity is influenced by only a few of them. This may give an indirect explanation to why spectral envelope is a stronger correlate to the perceived quality of pianos tone in discrimination tasks than inharmonicity.

\section{Conclusions}

In conclusion, the present study indicates that the low quality of bass tones of small pianos compared to the large pianos is determined more by the spectral envelope and its evolution, than by the time-invariant inharmonicity coefficient. In two listening tests, utilizing synthesized as well as real piano tones, it was clearly shown that the distinction between a bass tone of a small upright piano and that of a large grand piano can be attributed primarily to differences in spectral envelope. Inharmonicity played a secondary role only, despite a large variation range. Our results are at variance with a widespread conception based on old studies, emphasizing the importance of inharmonicity. Further support of our findings would need more experiments using high-fidelity piano synthesis.

Differences in the evolution of the spectral envelope would perceptually be related to the balance between low-frequency and high-frequency energy in spectrum, influencing the brightness (sharpness) of the tone. A possible candidate for quantifying the physical correlate of this perceptual effect would be the spectral centroid and its evolution during the decay of the piano tone. Spectral irregularity [38] is another envelope factor worth to investigate in further studies of the quality of piano tones.

\section{Acknowledgements}

This project was supported by a NATO Postdoctoral Science Fellowship awarded to the first author for visiting Queens's University, Kingston, Canada, followed by a visiting professorship grant from the Wenner-Gren Foundations for working at the Royal Institute of Technology $(\mathrm{KTH})$, Stockholm, Sweden. The authors are grateful to Sonya Dal Cin and Jung-Kyong Kim for running participants and data preparation. 


\section{References}

Footnote 1: The design of the experiments in the frequently cited study by Fletcher et al [6] has been heavily criticized in retrospect, and several of the results seem questionable $[9,31]$.

[1] A. Galembo, L. L. Cuddy: Large grand vs. small upright pianos: Factors of timbral difference. J. Acoust. Soc. Am. 102, Part 2 (1997) 3107.

[2] Music: Large Encyclopedic Dictionary (in Russian). Large Russian Encyclopedia Publishing House, Moscow, 1998.

[3] L. Fine : The Piano Book. Brookside Press, Boston MA, 1990, 14.

[4] W. V. McFerrin: The Piano - its acoustics. Tuners Supply Co., Boston MA, 1972, 155.

[5] O. Schuck, R. W. Young: Observation of the vibrations of piano strings. J. Acoust. Soc. Am. 15 (1943) 1-11.

[6] H. Fletcher, E. D. Blackham, R. Stratton: Quality of piano tones. J. Acoust. Soc. Am. 34 (1962) 749-761.

[7] K. Exley: Tonal properties of the pianoforte in relation to bass bridge mechanical impedance. J. Sound and Vibr. 9 (1969) 420-437.

[8] H. A., Jr. Conklin: Design and tone in the mechanoacoustic piano. Part 3: Piano strings and scale design. J. Acoust. Soc. Am. 100 (1996) 1286-1298.

[9] A. H. Benade: Fundamentals of Musical Acoustics. Oxford University Press, New York, 1976.

[10] A. Reinholdt, E. Jansson, A. Askenfelt: Analysis and synthesis of piano tone. J. Acoust. Soc. Am. 81, Suppl. 1 (1987) 46.

[11] A. Galembo, A. Askenfelt, L. L. Cuddy, F. Russo: Effects of relative phases on pitch and timbre in the piano bass range. J. Acoust. Soc. Am. 110 (2001) 1649-1666.

[12] P. Bilhuber, C. Johnson: Influence of the soundboard on piano tone quality. J. Acoust. Soc. Am. 11 (1940) 311-320.

[13] D. W. Martin: Decay rates of piano tones. J. Acoust. Soc. Am. 19 (1947) 535-541.

[14] T. C. Hundley, H. Benioff, D. W. Martin: Factors contributing to the multiple rate of piano tone decay. J. Acoust. Soc. Am. 64 (1978) 1303-1309.

[15] K. Wogram: Acoustical research on pianos. Part I: Vibrational characteristics of the soundboard. Das Musikinstrument 24 (1981) 694-702, 776-82, 872-80.

[16] K. Wogram: The string and the soundboard. - in Five lectures on the Acoustics of the Piano. A. Askenfelt (ed.). Royal Swedish Academy of Music, 1990, 83-94. 
[17] H. Suzuki, I. Nakamura: Acoustics of pianos. Applied Acoustics 30 (1990) 147-205.

[18] I. Nakamura: Characteristics of piano sound: Acoustical research on the piano. Part 4. J. Acoust. Soc. Jpn. (E) 17 (1996) 255-262.

[19] I. Bork: Grand piano and upright piano: Differences in sound and radiation. Proc. International Symposium on Musical Acoustics (ISMA'97), 19 - 22 August 1997, Edinburg, 1997, 79-84.

[20] N. Giordano: Sound production by a vibrating piano soundboard: Experiment. J. Acoust. Soc. Am. 104 (1998) 1648-1653.

[21] H. Fletcher: Normal vibration frequencies of a stiff piano string. J. Acoust. Soc. Amer. 36 (1964) 203-209.

[22] A. Galembo, L. L. Cuddy: String inharmonicity and the timbral quality of piano bass tones: Fletcher, Blackham, and Stratton (1962) revisited. Proc. $3^{\text {rd }}$ US Conference on Music Perception and Cognition, MIT, Cambridge (1997), p. 3.

[23] A. Galembo, A. Askenfelt: Representation of signals and estimation of spectral parameters by inharmonic comb filters with application to the piano. IEEE Transactions on Speech and Audio Processing 7 (1999) 197-203.

[24] W. H. Lichte: Attributes of complex tones. J. Exper. Psychol. 28 (1941) 455-480.

[25] G. v. Bismarck: Sharpness as an attribute of the timbre of steady sounds. Acustica 30 (1974) 159-172.

[26] E. Zwicker and H. Fastl: Psychoacoustics: Facts and Models. Springer-Verlag, New York, 1990.

[27] L. Demany, C. Semal: Pitch versus brightness of timbre: Detecting combined shifts in fundamental and formant Frequency. Music Perception 11 (1993) 219-142.

[28] SOUNDSWELL ${ }^{\mathrm{TM}}$ Signal Workstation, Hitech Development, Box 2058, SE-174 02 Sundbyberg, Sweden.

[29] SPRUCE Listening Test ${ }^{\mathrm{TM}}$, Hitech Development, Box 2058, SE-174 02 Sundbyberg, Sweden.

[30] P. Kleczkowski: Group additive synthesis. Computer Music Journal 13 (1989) 12-20.

[31] J. R. Lee: Digital Sound-Synthesis Algorithms: A Tutorial Introduction and Comparison of Methods. PhD dissertation, University of California, San Diego, 1988.

[32] J. R Lee: Method and apparatus for producing an electronic representation of a musical sound using extended coerced harmonics. US Patent No. 5,466,882 (Cl. 84/603) Gulbransen, Inc. (1995).

[33] D. Rocchesso, F. Scalcon: Bandwidth of perceived inharmonicity for physical modeling of dispersive string. IEEE Transactions on Speech and Audio Processing 7 (1999) 597-601. 
[34] S. McAdams, J. W. Beauchamp, S. Meneguzzi, S.: Discrimination of musical instrument sounds resynthesized with simplified spectrotemporal parameters J. Acoust. Soc. Am. 105 (1999) 882-897.

[35] Rimski-Korsakov, A., Diakonov, N. Musical Instruments: Methods of research and calculation. (in Russian) Rosgizmestprom Press, Moscow, 1952, 167.

[36] Askenfelt A. \& Jansson E. From touch to string vibrations I: Timing in the grand piano action, J. Acoust. Soc. Am. 88 (1990) 52-62.

[37] A. Galembo, L. Ivanovskaia: Spectral time structure and noise sources in the high treble range of the piano (in Russian). Proc. 9th USSR Acoustical Conference, Moscow (1977) 3740 .

[38] J. Krimphoff, S. McAdams, S. Winsberg: Caracterisation du timbre de sons complexes. II: Analyses acoustiques et quantification psychophysique. Journal de Physique, 4(C5) (1994) 625-628. 


\section{Figure legends}

Fig.1. Initial spectrum of a grand piano bass tone (Steinway $\mathrm{C}, \mathrm{A}_{0}=27.5 \mathrm{~Hz}$ ) recorded by a microphone at a distance of about $1 \mathrm{~m}$. Spectrum shows more than 100 partials extending to 4-5 kHz. The spectrum envelope has a characteristic formant-like shape with groups of about eight partials between spectral minima, determined by the striking position of the hammer. The partials are progressively stretched due to string inharmonicity.

Fig. 2. Spectra of four synthesized inharmonic piano-like tones $\left(A_{0}=27.5 \mathrm{~Hz}\right)$ showing the effect on spectral bandwidth due to the stretching of the partials. With equal number of partials $(N=100)$ a tone with high inharmonicity $\left(B=600 \cdot 10^{-6}\right)$ extends to a frequency about twice as high as the low-inharmonic tone $\left(B=60 \cdot 10^{-6}\right)$ (left pair). In order to match the spectral bandwidths only 66 partials are required in the high-inharmonic tone (right pair).

Fig. 3. Spectra of a synthesized inharmonic tone $\left(\mathrm{E}_{1}=41.2 \mathrm{~Hz}, B=267 \cdot 10^{-6}\right)$ with 48 partials $\left(W_{1,3}\right)$ showing the progressive decay of higher partials at three time points; at the onset (top), after $0.5 \mathrm{~s}$ (middle), and after $1.0 \mathrm{~s}$ (bottom)

Fig. 4. Mean scores of timbral difference ratings in Listening test 1. The effect of spectral bandwidth on timbral difference is far larger than the effect of inharmonicty.

Fig. 5. Initial spectra for the three $A_{0}$ tones compared in Listening test 2. Small upright píano, Nordiska Pianofabriken, model Futura $112 \mathrm{~cm}$ (recording, $B=560 \cdot 10^{-6}$ ) (top), hybrid tone (synthesized, $B=160 \cdot 10^{-6}$ ) (middle), and concert grand piano, Steinway model $\mathrm{D}$ (recording, $B=160 \cdot 10^{-6}$ ) (bottom).

Fig. 6. Mean scores and standard deviations (bars) for timbral difference ratings in Listening test 2 . The hybrid-upright tone pair (left) had the same spectral envelope, while the hybrid grand tone pair (right) had the same inharmonicity. 